\title{
Impact of Controlling Nutritional Status Score (CONUT) and Prognostic Nutritional Index (PIN) on Patients Undergoing Coronary Artery Bypass Graft Surgery
}

\author{
Melike Elif Teker Açıkel, ${ }^{1}$ Ali Kubilay Korkut ${ }^{2}$ \\ ${ }^{1}$ Department of Cardiovascular Surgery, Haseki Training and Research Hospital, Istanbul, Turkey; ${ }^{2}$ Department of Cardiovascular \\ Surgery, Haliç University, Istanbul, Turkey
}

\section{ABSTRACT}

Background: The aim of this study is to evaluate the negative effect of malnutrition in patients with coronary artery disease who are undergoing coronary artery bypass graft surgery.

Methods: In this study, we analyzed 149 patients, who underwent coronary artery bypass surgery. Nutritional status of the patients was classified using controlling nutritional status score (CONUT) and prognostic nutritional index (PNI). Statistical correlation between malnutrition and complication following operation was evaluated with the chi-square test. Statistical alpha significant level was accepted $P<0.05$.

Results: There were various complications in 38 patients. Renal failure was the predominant problem in 18 of them. There was statistical significance between malnutrition and complication $(P<.001)$. There were more complications in the controlling nutritional status score and prognostic nutritional index groups. Renal complication $(P<.001)$, hemorrhage $(P<.05)$, and mortality $(P<.05)$ were high in the severe controlling nutritional status score and prognostic nutritional index groups.

Conclusion: There are manifest correlations between the severe controlling nutritional status score and prognostic nutritional index groups and morbidity and mortality after coronary artery bypass graft surgery. We found that renal complications, hemorrhage, and mortality rates increase significantly in patients with severe malnutrition.

\section{INTRODUCTION}

Coronary artery disease (CAD) is a main cause of mortality worldwide; it increases with obesity and its associated co-morbidities. Coronary artery bypass graft (CABG) surgery still is the best treatment for multi-vessel and left main disease when considering the survival, improved ventricular function, freedom from recurrent angina, and reintervention

Received April 4, 2019; received in revised form May 23, 2019; accepted fune 6, 2019.

Correspondence: Melike Elif Teker Açıel, Department of Cardiovascular Surgery, Haseki Training and Research Hospital, Istanbul, Turkey; (e-mail: eteker@biruni.edu.tr).
[Taggart 2006]. Malnutrition is a serious problem in public health with prevalence rates between $30 \%$ to $70 \%$ in hospitalized patients [Kamath 1986], which has a bad influence on the cardiovascular system, immunological system, endocrine system, gastrointestinal system, and on the healing process during the recovery period [Cederhdm 1993].

Malnutrition is widespread among patients with cardiovascular diseases and is related to adverse postoperative outcomes. Furthermore, malnutrition occurs in $10 \%$ to $25 \%$ of patients undergoing cardiac surgery [Van Venrooji 2008]. Several different tools have been developed for nutritional screening. Controlling nutritional status score (CONUT) and prognostic nutritional index (PNI) are the most widely used tools.

In this study, we evaluate the negative effect of malnutrition using nutritional screening scores CONUT and PNI on patients undergoing CABG surgery.

\section{MATERIALS AND METHODS}

The present study included 149 patients (>18 years) undergoing CABG between May 2017 and May 2018. Among these patients, 119 (79.9\%) were male and 30 (20.1\%) female. Their ages ranged between 19 years to 79 years (mean 59.1). There were associated diseases, including diabetes mellitus in $51(34.2 \%)$ patients, hypertension in $54(36.2 \%)$, peripheral arterial disease in seven $(4.7 \%)$, chronic renal failure in six $(4.0 \%)$, and miscellaneous systemic diseases in $25(16.8 \%)$ patients. Demographical data of the patients are shown on Table 1.

The patients were classified using CONUT and PNI, according to their nutritional status and nutritional risk. The calculation of CONUT is serum albumin $(\mathrm{g} / \mathrm{dL})+$ total lymphocyte count (per mL) + total cholesterol (mg/ $\mathrm{dL})$. The calculation of PNI is $10 \mathrm{x}$ serum albumin $(\mathrm{g} / \mathrm{dL})$ $+0.005 x$ total lymphocyte count (per mL). Both CONUT and PNI scores were used as nutritional predictors. The patients were classified into three different malnutrition groups; they were named mild, moderate, and severe. PNI and CONUT scores of the patients were calculated and presented on Table 2 .

Postoperative complications were defined complications observed during the hospital stay following bypass surgery. 
Table 1. Demographical Data of Patients

\begin{tabular}{llcc}
\hline & & Mean \pm SD & Min-Max \\
\hline Ages & $59.1 \pm 10.3$ & $19-79$ \\
\hline \multirow{2}{*}{ Sex } & & N & $\%$ \\
\hline \multirow{3}{*}{ Associated Diseases } & & 119 & 79.9 \\
& & 30 & 20.1 \\
& Demale & 119 & 79,9 \\
& Hiabetes Mellitus & 51 & 34.2 \\
& Coronary Artery Disease & 81 & 55.1 \\
& Peripheral Arterial Disease & 7 & 4.7 \\
& Chronic Renal Failure & 6 & 4.0 \\
& Other Systemic Diseases & 25 & 16.8
\end{tabular}

These complications included hemorrhage, renal, neurological, and pulmonary problems. Mortality was defined as in-hospital mortality following bypass operation. The study protocol was approved by the S.B.Ü.IstanbulTraining and Research Hospital Ethics Committee. The study was conducted in accordance with the principles of the Declaration of Helsinki.

Table 2. PNI and CONUT Index of Patients

\begin{tabular}{lcc}
\hline & Mean \pm SD & Min-Max (Median) \\
\hline PNI & $47.6 \pm 6.8$ & $33-65,85$ (47.2) \\
\hline & $\mathrm{N}$ & $\%$ \\
\hline$<46.7$; severe & 69 & 46.3 \\
46.7-50.8; medium & 35 & 23.5 \\
$>$ 50.8; mild & 45 & 30.2 \\
\hline & Mean \pm SD & Min-Max (Median) \\
\hline CONUT & $1.70 \pm 1.66$ & $0-6(1)$ \\
\hline
\end{tabular}

\begin{tabular}{lll}
\hline & $\mathrm{N}$ & $\%$ \\
\hline 0-1; mild & 80 & 53.7 \\
2; medium & 27 & 18.1 \\
$>$ 3; severe & 42 & 28.2
\end{tabular}

\section{STATISTICAL ANALYSIS}

Data obtained in the study were analyzed using the SPSS version 15.0 software (SPSS Inc., Chicago, IL, USA). Descriptive statistical analysis results were shown as mean \pm standard deviation values for numerical data, and as number $(\mathrm{N})$ and percentage (\%) for categorical data. Baseline characteristics were compared using the Fisher Exact Test, Pearson Chi-Square test and the Independent Samples t-test, as appropriate. Comparisons of CONUT and PNI complications were made using the Fisher's Exact Test and Pearson Chi-square analysis. Statistical significance was accepted as $P<.05$.

\section{RESULTS}

Various complications were seen after CABG surgery in $38(25.5 \%)$ patients. Renal failure was the most predominant issue in $18(12.1 \%)$ of them. Hemorrhage occurred with eight $(5.4 \%)$ patients. Pulmonary problems were seen in five $(3.4 \%)$, and neurological troubles were seen in three $(2.0 \%)$ patients. Mortality was seen in seven $(4.7 \%)$ patients. All complications are shown on Table 3.

In the PNI groups, there was statistical significance between malnutrition and complication $(P<.001)$. More complications occurred with the severe PNI group. Renal complication $(P<.001)$, hemorrhage $(P=.038)$, and mortality $(P=.025)$ were high in the severe PNI group. The correlation between PNI and postoperative complications is shown on Table 4.

In the CONUT groups, there was statistical significance between malnutrition and complication $(P<.001)$. More complications occurred in the severe CONUT group. Renal complication $(P<.001)$, hemorrhage $(P=.007)$, and mortality $(P=.005)$ were high in the severe CONUT group. The correlation between CONUT and postoperative complications is shown on Table 5 .

Table 3. Complications after CAGB Surgery (NA not available, A available)

\begin{tabular}{lll}
\hline & N & $\%$ \\
\hline Complication & & \\
NA & 111 & 74.5 \\
A & 38 & 25.5 \\
Renal & 18 & 12.1 \\
Neurological & 3 & 2.0 \\
Hemorrhage & 8 & 5.4 \\
Pulmonary & 5 & 3.4 \\
Mortality & 7 & 4.7
\end{tabular}


Table 4. Correlation Between PNI Groups and Postoperative Complications

\begin{tabular}{|c|c|c|c|c|c|c|c|c|c|c|c|c|}
\hline $\mathrm{PNI}$ & $\mathrm{N}$ & $\%$ & $N$ & $\%$ & $N$ & $\%$ & $\mathrm{~N}$ & $\%$ & $\mathrm{~N}$ & $\%$ & $N$ & $\%$ \\
\hline Medium & 3 & 8.6 & 1 & 2.9 & 0 & 0.0 & 1 & 2.9 & 1 & 2.9 & 0 & 0.0 \\
\hline Mild & 2 & 4.4 & 0 & 0.0 & 1 & 2.2 & 0 & 0.0 & 1 & 2.2 & 0 & 0.0 \\
\hline
\end{tabular}

Table 5. Correlation Between CONUT Groups and Postoperative Complications

\begin{tabular}{|c|c|c|c|c|c|c|c|c|c|c|c|c|}
\hline CONUT & $N$ & $\%$ & $\mathrm{~N}$ & $\%$ & $\mathrm{~N}$ & $\%$ & $N$ & $\%$ & $N$ & $\%$ & $N$ & $\%$ \\
\hline Medium & 6 & 22.2 & 2 & 7.4 & 0 & 0.0 & 1 & 3.7 & 1 & 3.7 & 2 & 7.4 \\
\hline Severe & 28 & 66.7 & 16 & 38.1 & 2 & 4.8 & 6 & 14.3 & 2 & 4.8 & 5 & 11.9 \\
\hline
\end{tabular}

\section{DISCUSSION}

There is an association between low PNI, which is a marker based on the nutritional-inflammatory process based on serum albumin levels and the lymphocyte count. Low PNI levels correlate with increased mortality rates in CAD patient following CABG surgery [Keskin; Wada 2018]. In our study, we determined significant statistical correlations between the severe PNI group and increased postoperative renal complication, hemorrhage, and mortality following CABG surgery.

There are studies about the correlation between a high CONUT score, a marker of poor nutrition, and increased complication rates following percutaneous coronary intervention in stable CAD patients [Wada 2017].

Atherosclerotic plaque rupture is a lymphocyte-mediated inflammatory process [ $\mathrm{Pu} 2012]$.

In the acute period of the CAD, lymphocytopenia is a common finding during the stress response and is secondary to increased corticosteroid levels. On the other hand, lymphocytosis is considered a more appropriate inflammatory process and quiescent of the inflammatory status [Onsrud 1981]. There are studies about correlation between lymphocytopenia and occurrence of $\mathrm{CAD}$ complications and mortality following myocardial infarction [Arbel 2012]. Serum albumin is an inhibitor of platelet aggregation that increases the production of the prostaglandin D2 (PGD2), which plays a major role in anti-aggregation [Maalej 1999]. In addition, hypoalbuminemia increases blood viscosity and disrupts endothelial function [Joles 1997].

Malnutrition has been recognized as an independent risk factor for morbidity and mortality in patients with chronic heart failure (CHF) [Kalantar-Zadeh 2004; Sargento 2014]. The prognostic influence of malnutrition in CAD is not very well understood. Decreases of the serum albumin level may increase risk factors for CAD [Oduncu 2013].
Our study indicates that PNI and CONUT are significant prognostic factors for CAD patients undergoing CABG surgery. There are manifest correlations between the severe PNI and CONUT groups and morbidity and mortality after CABG surgery. We found that renal complications, hemorrhage, and mortality rates increase significantly in patients with severe malnutrition.

\section{REFERENCES}

Arbel Y, Finkelstein A, Halkin A, Birati EY, Revivo M, Zuzut M et.al. 2012. Neutrophil/lymphocyte ratio is related to the severity of coronary artery disease and clinical outcome in patients undergoing angiography. Atherosclerosis 225 (2); 456-460.

Cederhdm J, Jagren C, Hellström K. 1993. Nutritional status and performance capacity in internal medicine patients. Clin Nutr 12: 8-14.

Joles JA, Willekes-Koolschijn N, Koomans HA. 1997. Hypoalbuminemia causes high blood viscosity by increasing red cell lysophosphatidylcholine. Kidney international 52; 761-770.

Kalantar-Zadeh K, Block G, Horwich T, Fonarow G.C. 2004. Reverse epidemiology of conventional cardiovascular risk factors in patients with chronic heart failure. J American College of Cardiology. 43; 1439-1444.

Kamath SK, Lawler M, Smith AE, Kalat T, Olson R. 1986. Hospital malnutrition: a 33-hospital screening study. J Am Diet Assoc 86: 203-206.

Keskin M, Ipek G, Aldag M, Altay S, Hayiroglu MI, Borklu EB, et.al. 2017. Effect of nutritional status on mortality in patients undergoing coronary artery bypass grafting. Nutrition. 48: 82-86.

Maalej N, Albrecht R, Loscalzo J, Folts JD. 1999. The potent platelet inhibitory effects of S-nitrosated albumin coating of artificial surface. Journal of the American College of Cardiology 33; 1408-1414.

Oduncu V, Erkol A, Karabay C, Kurt M, Akgün T, Bulut M. et.al. 2013. 
The prognostic value of serum albumin levels on admission in patients with acute ST-segment elevation myocardial infarction undergoing a primary percutaneous coronary intervention. Coronary artery disease. 24 (2); 88-94.

Onsrud M, Thorsby E. 1981. Influence of in vivo hydrocortisone on some human blood lymphocyte subpopulations. I. Effect on natural killer cell activity. Scandinavian journal of immunology 13 (6); 573-579.

Pu J, Mintz GS, Brilakis ES, Banerjee S, Abdel-Karim AR, Maini B et.al. 2012. In vivo characterization of coronary palques: novel findings from comparing greyscale and virtual histology intravascular ultrasound and near-infrared spectroscopy. European Heart Journal. 33 (3); 372-383.

Sargento L, Longo S, Lousanda N, dos Reis R.P. 2014. The importance of assessing nutritional status in elderly patients with heart failure. Current heart failure reports. 11; 220-226.
Taggart DP, Thomas BF. 2006. Coronary artery bypass grafting is still the best treatment for multivessel and left main disease, but patients need to know. Ann Thorac Surg 82: 1966-1975.

Van Venrooji LM, van Leeuwen PA, de Vos R, Borgmeijer-Hoelen MM, de Mol BA. 2008. Postoperative protein and energy intake and postoperative complications in well-nourished, non-hospitalized elderly cardiac surgery patients. Clin Nutr 27: 117-121.

Wada H, Dohi T, Miyauchi K, Doi S, Konishi H, Naito R, et.al. 2017. Prognostic impact of nutritional status assessed by the controlling nutritional status score in patients with stable coronary artery disease undergoing percutaneous coronary intervention. Clin. Res. Cardiol 106: 875-883.

Wada H, Dohi T, Miyauchi K, Jun S, Endo H, Doi S, et.al. 2018. Relationship between the prognostic nutritional index and long-term clinical outcomes in patients with stable coronary artery disease. J. Cardiol. 72 (2): $155-161$. 